\title{
The Guru-UKM Method: Synergistic Effect of Hydrogel, Hydrofibre and Dermal Conservation in Burn Wound Management
}

\author{
Farrah-Hani Imran ${ }^{a}$, Chik lan $^{a}$, Enda Gerard Kelly ${ }^{b}$, Razman Jarmin $^{a}$ \\ ${ }^{a}$ Department of Surgery, Universiti Kebangsaan Malaysia Medical Centre (UKMMC), Jalan Yaacob Latiff, \\ Bandar Tun Razak, 56000, Cheras, Wilayah Persekutuan Kuala Lumpur, Malaysia \\ ${ }^{b}$ Cappagh National Orthopaedic Hospital, Finglas, Dublin 11, Ireland
}

\section{ABSTRACT}

Initial wound care idioms were designed around a moist dressing in presumed better wound healing. As wound care advances, innovations of dressings were formed. In the Guru-UKM Method (GUM), we combined two well-established dressings producing a synergistic effect in burn wound management. Patients with deep partial thickness burns were selected for the GUM. From the time of admission, they receive 2 cycles of paraffin tulle dressings once every two days to allow demarcation, then are reassessed for suitability of the GUM technique. We discuss 7 different burn cases that presented to our Burn Unit from January 2014 - June 2015.All dressings should create a suitable moist environment for healing, yet should be a painless dressing to help the patient return to normal function as soon as possible. In burn wounds, a suitable dressing ideally also biochemically debrides fibrin and softens hardened eschar and slough, without necessitating the patient to undergo general anaesthesia and surgical debridement. The Guru-UKM Method is a combination dressing technique that facilitates optimal burn wound management.

KEYWORDS: Hydrogel, hydrofibre, burn wounds, debridement, dermal conservation

\section{INTRODUCTION}

Winter $^{1}$ (1962) introduced the concept of moist dressings to practice with a description of progressive wound healing in a moist environment compared with a dry dressing approach. Current advanced wound care dressings target different properties that particular dressing types have to enhance wound healing.

As technology advanced, new types of dressings were developed, including hydrogel, hydrocolloid and hydrofibre. Hydrogel is a water- or glycerinbased dressing consisting of water in a nonadherent, cross-linked polymer, which not only

Corresponding author:

Farrah-Hani Imran

Head of Plastic \& Reconstructive Surgery,

Burn Unit \& Wound Care Team,

Department of Surgery, Faculty of Medicine, Universiti Kebangsaan Malaysia Medical Centre

(National University of Malaysia),

Jalan Yaakob Latiff, 56000, Kuala Lumpur,

Malaysia

Tel: +603-91456202

Email: farrahhani@gmail.com keeps a wound well moisturized, but has an autolytic properties. Hydrocolloid are hydrophilic colloid particles bound to polyurethane foam that absorbs fluids while providing enough moisture to the wound bed. Its properties also include impermeable to bacteria and other contaminants ${ }^{2}$. Hydrofibre is a highly absorbent dressing, consisting of sodium carbomethylcellulose that interacts with wound exudate to form a gel that does not need to be changed frequently ${ }^{2}$.

With the development of new dressings based on clinical evidence, combination therapy was attempted to optimise wound healing. We present our case series using the combination of two established types of wound care dressing, hydrogel and hydrofibre. At our centre, the hydrogel used is DuoDERM ${ }^{\circledR}$ Hydroactive ${ }^{\circledR}$ Sterile Gel (Convatec Inc.), which has hydrocolloid properties, and the hydrofibre is Aquacel $\otimes_{A g}$ BURN Hydrofiber $\circledast$ dressing (ConvatecInc.)

This method was invented in the Burns Unit of UKM Medical Centre and subsequently applied to cases 
referred to the UKMMC Burns Unit \& Wound Care Team. The dressing is kept 3-5 days per cycle depending on the daily inspection of the dressing. Full-thickness burn wounds are excluded from this technique as full-thickness wounds generally require formal surgical debridement and subsequent wound bed preparation.

\section{METHODOLOGY}

Patients with wounds that were referred to the UKMMC Burns Unit \& Wound Care Team for wound bed preparation and management were assessed for suitability to use the Guru-UKM Method (GUM).

Deep partial-thickness burn wounds with a high degree of fibrinous slough were recruited as candidates for this method.

On presentation to our unit at the post-burnday (PBD) 1, the wound is assessed, blisters de-epithelialized, then dressed with Paraffin Tulle
(PT) dressing once every 2 days for 2 cycles to facilitate demarcation of severity. If suitable for GUM, the wound is first cleaned with normal saline, then hydrogel applied as a primary layer (as an interface between the wound bed and hydrofibre), followed by dry gauze and crepe bandage. The dressing is kept for 3-5 days before the next wound inspection (WI), unless there is strike-through, in which the outer layer is changed. If the hydrofibre is soaked, the entire dressing is replaced.

Non-viable tissue is removed by mechanical debridement during hydrotherapy at each serial wound assessment. Throughout the active wound management, the patients are given aggressive physiotherapy and reviewed by the dietician and an occupational therapist. Preexisting comorbidities are optimized by multi-disciplinary team management. Upon epithelization, they are advised on scar management including compliance to pressure garments.

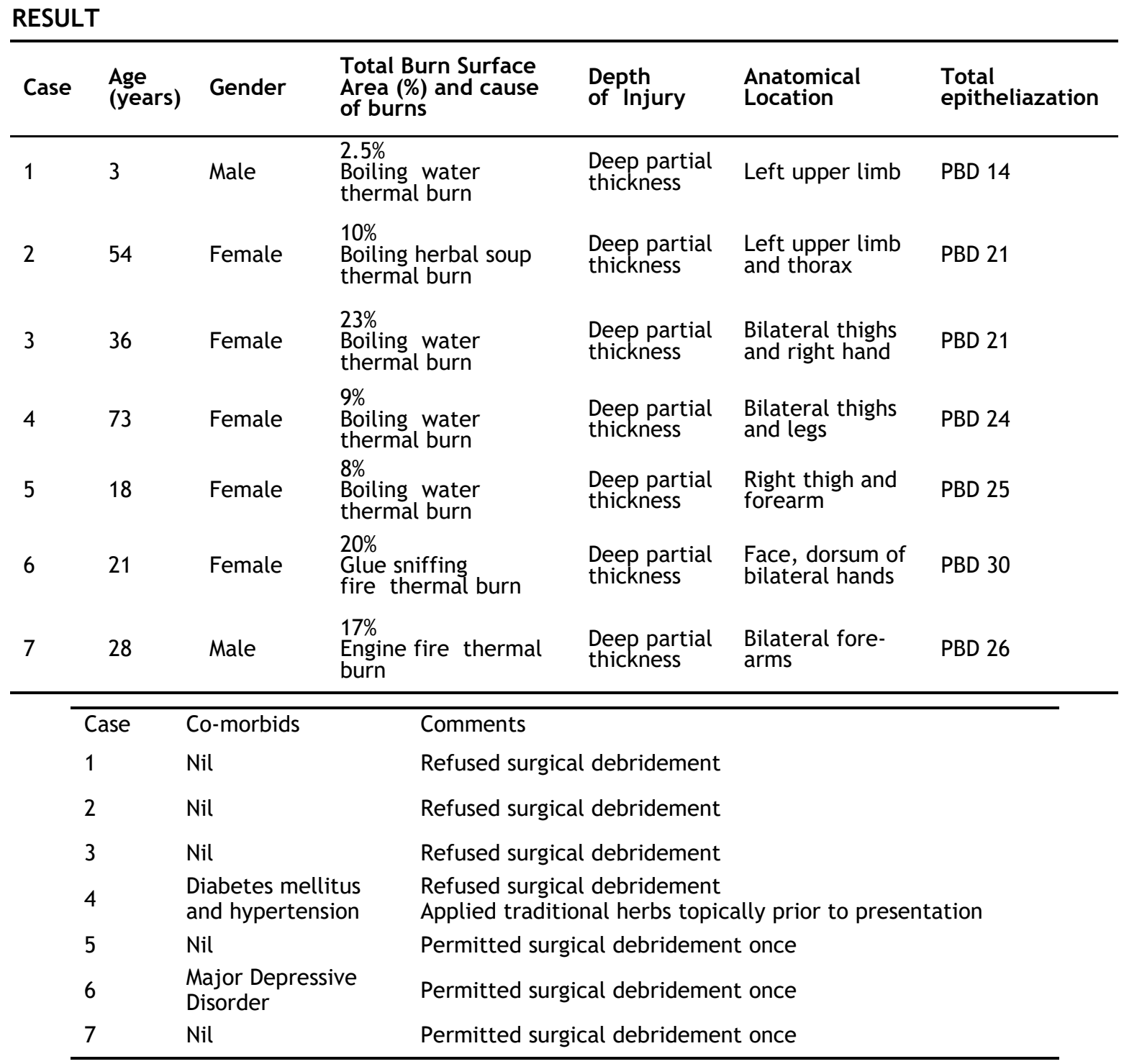


The progression of wound healing of case 5 is shown in Figure 1 to 3. By PBD 7 (Figure 1), the patient showed steady gradual response to GUM. At each dressing change, remaining non-viable tissue was gently peeled away and easily removed painlessly (Figure 2). Her wounds were epithelialised by PBD 25 (Figure 3).

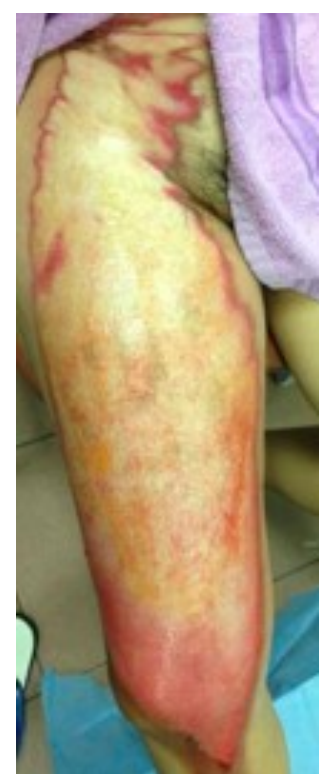

Figure 1: Post burn day 7 (right thigh) of case 5

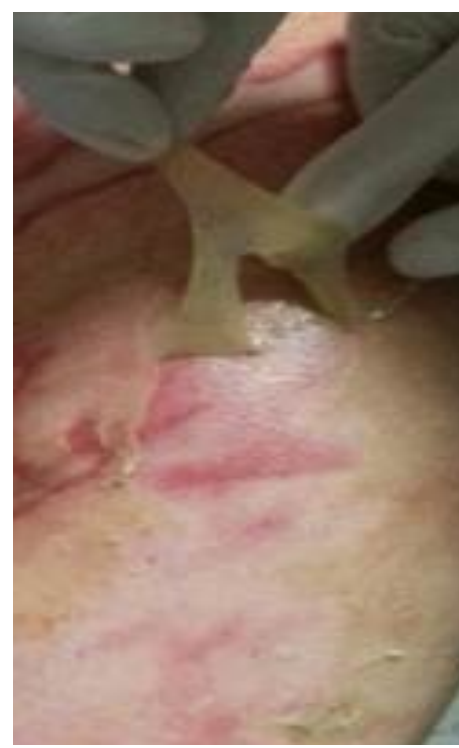

Figure 2: demonstration of easy and painless removal of non-viable tissue softened by the Guru-UKM Method.

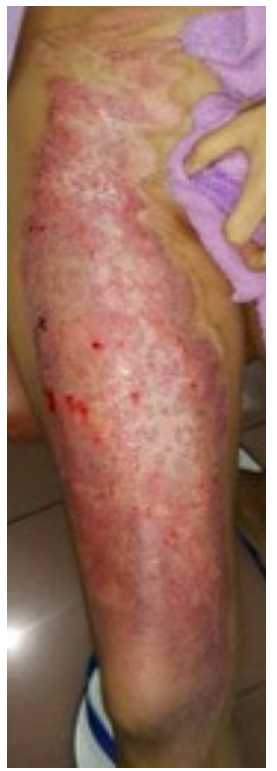

Figure 3: Post burn day 21 (right thigh) of case 5

\section{DISCUSSION}

Hydrofibre is important in creating a moist environment. However, it does not provide autolytic debridement, which may be useful in burn wounds that form slough. Hydrogel added into the dressing can act as both a water donator and absorber, also keeping the wound bed moist while chemically desloughing the wound ${ }^{3}$. As it is a gel, it would require a secondary dressing to keep it in place. Instead of traditional gauze, (which may absorb fluid initially, but leak fluid when it's full) the use of hydrofibre keeps the hydrogel in place and moderates the moisture. It functions to prevent maceration of the wound by absorbing extra moisture and does not allow excessive loss of fluid laterally, maintaining it at the wound. When a hydrofibre dressing has absorbed water, it turns into a gel and conforms to the wound, preventing bacteria to proliferate in the wound space ${ }^{4}$. It is also important to note that the hydrofibre used is lined with silver, which is an important antimicrobial that lasts up to 14 days $^{5}$.

In this case series, patients are dressed with this 2 layered technique. As burn wounds form a fibrinous slough that prevents natural granulation and subsequent wound healing, patients with partial thickness wounds traditionally may be subjected to surgical debridement by tangential excision under general anaesthesia, depending on the depth and zone of injury. Mechanical debridement can be painful and traumatizing to the patient, and is an important factor of consideration of dressing; especially as burn wounds require regular assessment. Surgical debridement by inexperienced hands may end up removing more tissue than necessary, excising healthy dermal tissue, further complicating and delaying the healing process.

In conservative management, patients undergoing traditional dressing management may face collateral wounds, developing secondary to maceration of surrounding skin due to excess fluid. With the GUM, the hydrofibre does not adhere to the wound and is painless on removal as compared to traditional gauze $^{6}$. Therefore, patient comfort during dressing change is optimized while maintaining healthy granulation tissue and neo-epithelialization. Previous literature,$^{7,8}$ has assessed silver sulfadiazine dressing to hydrofibre and demonstrated where patients felt there was less pain during the removal and patients were less anxious during dressing. 
The basis of any dressing is to facilitate wound healing, to prevent infection and should be of minimal pain to the patient pre, during and post dressing. This method was borne out of necessity due to dealing with patients refusing surgical debridement or general anaesthesia for personal, cultural or religious reasons. This is a common occurrence in multi-ethnic and multi-cultural patient cohort presenting to our centre. We realized we must be able to provide a reliable dressing regime based on existing clinical evidence that deals with the challenges of a burn wound while respecting the patient's wishes for conservative management.

It must be emphasized that GUM is not a substitute for surgical debridement and/or skin grafting in fullthickness burns. We are also not endorsing a single one-size-fits-all dressing approach to burn wounds. Wound management is a dynamic process, with wounds usually requiring different dressing modalities as it progresses through the different stages of wound healing. Of these 7 cases, GUM was the solitary dressing method used successfully.

GUM facilitates gradual mechanical debridement when a patient refuses surgical debridement under anaesthesia. In addition, we found that by implementing GUM, the concept of dermal conservation is achieved. Healthy dermis, which might be inadvertently excised during tangential excision is preserved. At each wound inspection, non-viable tissue is removed by mechanical debridement and hydrotherapy.

GUM also softens hardened slough and eschar. This permits efficient debridement as the non-viable tissue is broken down and more amenable to effective excision and debridement, be it surgically or mechanically. In the 3 cases that agreed for a singular surgical debridement, the residual plaques of eschar and slough were easily curetted off, similar to softened butter, with minimal trauma to underlying healthy dermis.

Once the bulk of non-viable tissue is removed, patients could have been discharged \& return for wound inspection and dressing at our outpatient dressing clinic. However, most patients prefer to remain as inpatients due to lack of social support. As inpatients, they are managed by the multidisciplinary UKMMC Burns Unit Team consisting of plastic surgery, specialized nursing, physiotherapy, occupational therapy, dietician \& nutritional support, psychology, acute pain service, infectious diseases, paediatrics and others as necessary. Although total costing for treatment isn't calculated in this series, Yarboro et $\mathrm{al}^{8}$ have shown the cost of modern dressings (hydrofibre) are comparable to long established dressings (silver sulfadiazine). In addition, Verbelen et $\mathrm{al}^{9}$ have shown that hydrofibre silver dressing is comparable or superior to a wellknown, frequently used nanocrystalline silver dressing. GUM has no restrictive properties and eases dressing changes in deep partial thickness burn wounds by reducing pain, painless removal of eschar, slough and the hydrofibre layer, requiring less or no analgesia.

In our case series, our patients required 18-30 days to achieve full epithelialization under GUM. Three cases had a single wound debridement under general anaesthesia, whereby GUM had made the debridement easier and less traumatic. The next step would be to perform a randomized control trial and comparison studies to further evaluate the efficacy of GUM.

\section{CONCLUSION}

This is a combination dressing technique that facilitates optimal burn wound management in patients requesting conservative management. We found that clinically, GUM successfully achieves simultaneous debridement and epithelialization in deep partial thickness burn wounds while preserving uninjured dermal tissue within the zone of injury. Further studies will be performed to eradicate any bias and fully evaluate its clinical significance.

\section{REFERENCES}

1. George D Winter. Formation of the Scab and the Rate of Epithelization of Superficial Wounds in the Skin of the Young Domestic Pig. Nature (20 January 1962) 193,293-294

2. Baranoski S. WOUND AND SKIN CARE: Choosing a wound dressing, part 2; Nursing, February 2008; 38(2):14-15.

3. Stephen Thomas, Paul Hay. Fluid handling properties of hydrogel dressings. Ostomy/ Wound management, April 1995;41(3):54-6, 589.

4. Samantha Jones, Philip G. Bowler, Mike Walker. 
Antimicrobial Activity of Silver-Containing

Dressings is Influenced by Dressing

Conformability with a Wound Surface. Wounds

2005;17(9):263-270.

5. Bowler PG, Jones SA, Walker M, Parsons D.

Microbicidal properties of a silver-containing hydrofibre dressing against a variety of burn wound pathogens. J Burn Care Rehabil, Mar-Apr 2004;25(2):192-196.

6. Hoekstra MJ, Hermans MH, Richters CD, Dutrieux RP. A histological comparison of acute inflammatory responses with a hydrofibre or tulle gauze dressing. J Wound Care, Mar 2002;11(3):113-117.

7. Daniel M. Caruso, Kevin N. Foster, Sigri A. Blome-Eberwein, John A. Twomey, et al. Randomized Clinical Study of Hydrofibre Dressing With Silver or Silver Sulfadiazine in the Management of Partial-Thickness Burns. Journal of Burn Care \& Research, May-Jun 2006; 27(3): 298-309.

8. Douglas D. Yarboro. A Comparative Study of the Dressings Silver Sulfadiazine and Aquacel Ag in the Management of Superficial Partial-

Thickness Burns. Advances in Skin \& Wound Care, Jun 2013; 26(6): 259-262.

9. Jozef Verbelen, Henk Hoeksema, Alexander Heyneman, Ali Pirayesh et al. Aquacel Ag dressing versus Acticoat dressing in partial thickness burns: A prospective, randomized, controlled study in 100 patients. Part 1: Burn wound healing. Burns, May 2014; 40(3) 416 427. 
